\title{
Identification of the optical and near-infrared counterpart of GRS 1758-258
}

\author{
A. J. Muñoz-Arjonilla ${ }^{1,2}$, J. Martí1,2, P. L. Luque-Escamilla ${ }^{3,2}$, J. R. Sánchez-Sutil ${ }^{2}$, E. Sánchez-Ayaso ${ }^{1,2}$, \\ J. A. Combi ${ }^{4}$, and I. F. Mirabel ${ }^{5}$
}

1 Departamento de Física, EPS, Universidad de Jaén, Campus Las Lagunillas s/n, Edif. A3, 23071 Jaén, Spain e-mail: [ajmunoz; jmarti; esayaso]@ujaen.es

2 Grupo de Investigación FQM-322, Universidad de Jaén, Campus Las Lagunillas s/n, Edif. A3, 23071 Jaén, Spain e-mail: jrssutil@ujaen.es

3 Dpto. de Ing. Mecánica y Minera, EPS, Universidad de Jaén, Campus Las Lagunillas s/n, Edif. A3, 23071 Jaén, Spain e-mail: peter@ujaen.es

4 Instituto Argentino de Radioastronomía (CCT La Plata, CONICET), C.C.5, (1894) Villa Elisa, Buenos Aires, Argentina e-mail: jcombi@fcaglp.unlp.edu.ar

5 Laboratoire AIM, IRFU/Service d'Astrophysique, Bat. 709, CEA-Saclay, 91191 Gif-sur-Yvette Cedex, France; Instituto de Astronomía y Física del Espacio (CONICET-UBA), CC 67, Suc. 28, 1428 Buenos Aires, Argentina e-mail: felix.mirabel@cea.fr

Received 5 February 2010 / Accepted 25 April 2010

\section{ABSTRACT}

\begin{abstract}
Context. Understood to be a microquasar in the Galactic center region, GRS 1758-258 has not yet been unambiguously identified to have an optical/near-infrared counterpart, mainly because of the high absorption and the historic lack of suitable astrometric stars, which led to the use of secondary astrometric solutions. Although it is considered with 1E 1740.7-2942 as the prototypical microquasar in the Galactic center region, the Galactic origin of both sources has not yet been confirmed.

Aims. We attempt to improve previous astrometry to identify a candidate counterpart to GRS $1758-258$. We present observations with the Gran Telescopio de Canarias (GTC), in which we try to detect any powerful emission lines that would infer an extragalactic origin of this source.

Methods. We use modern star catalogues to reanalyze archival images of the GRS 1758-258 field in the optical and near-infrared wavelengths, and compute a new astrometric solution. We also reanalyzed archival radio data of GRS 1758-258 to determine a new and more accurate radio position.

Results. Our improved astrometric solution for the GRS 1758-258 field represents a significant advancement on previous works and allows us to identify a single optical/near-infrared source, which we propose as the counterpart of GRS 1758-258. The GTC spectrum of this source is however of low signal-to-noise ratio and does not rule out a Galactic origin. Hence, new spectral observations are required to confirm or discard a Galactic nature.
\end{abstract}

Key words. X-rays: stars - radio continuum: stars - infrared: general - X-rays: binaries

\section{Introduction}

GRS $1758-258$ is one of the two brightest persistent hard X-ray sources in the Galactic center region and belongs to the class of Galactic microquasars (see e.g., Mirabel \& Rodríguez 1999, for a general discussion of microquasar properties). Originally discovered in hard X-rays (Sunyaev et al. 1991; Goldwurm et al. 1994), GRS 1758-258 was classified as a microquasar after the detection of radio bipolar jets emanating from it, extending over $\sim 1^{\prime}$ and ending with the formation of two radio lobes (Rodríguez et al. 1992). Although it is considered, together with 1E 1740.7-2942 (Mirabel et al. 1992), the prototypical microquasar in the Galactic center region, its morphology is reminiscent of a Fanaroff-Riley II galaxy and an extragalactic origin cannot be ruled out.

The radio source proposed as the exciting core of the GRS 1758-258 microquasar lobes is found to coincide with a Chandra X-ray source (Heindl \& Smith 2002; Martí et al. 2002). These X-ray and radio observations provide us with a very accurate sub-arcsec position, which is essential to find an optical/near-infrared counterpart in the crowded fields of the Galactic center region. Unfortunately, efforts made in this sense have been unable to unambiguously propose a single source as the possible counterpart to GRS 1758-258, mainly due to the absorption towards the Galactic center region and the early lack of suitable astrometric standards (Martí et al. 1998; Rothstein et al. 2002).

In this work, we have taken advantage of the existence of modern star catalogues, which allow us to improve the astrometry of the GRS 1758-258 field in the optical and near-infrared wavelengths. We also present a new and more accurate radio position of this microquasar that we derived based on a revision of archival data. As a result, we find that there is only one optical/infrared object astrometrically coincident with the accurate GRS 1758-258 X-ray and radio positions, which we propose 


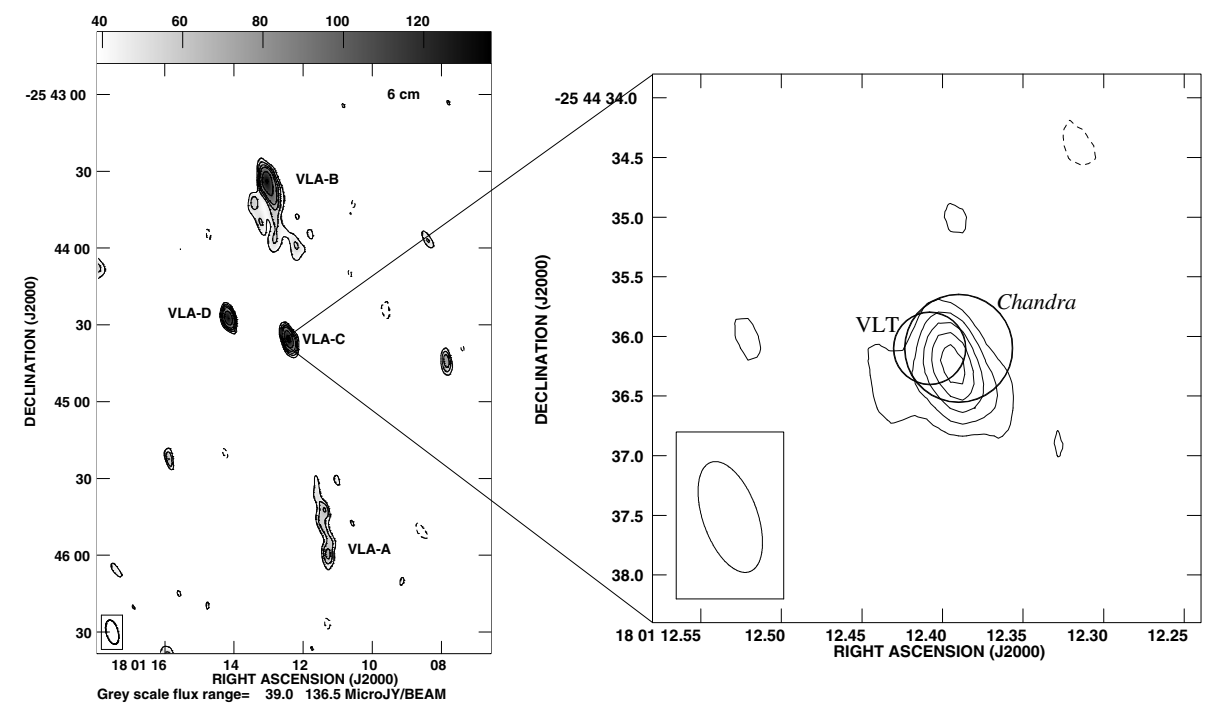

Fig. 1. Left. Large-scale radio map of GRS 1758-258 at $6 \mathrm{~cm}$ wavelengths (adapted from Martí et al. 2002). The central core of the microquasar is labelled VLA-C. The collimated jets end with the formation of two radio lobes (VLA-A and VLA-B). VLA-D is considered to be a likely non-related radio source. Right. Central core of GRS 1758-258 at $6 \mathrm{~cm}$, obtained in this work after combining all the A-configuration VLA data in Table 1. Radio contours correspond to $-3,3,5,7,9$, and 11 times the rms noise of $30 \mu \mathrm{Jy}$. The synthesized beam of this high resolution map is shown at the bottom left corner, its size being $00^{\prime \prime} 97 \times 0^{\prime \prime} 46$ with a position angle of $19{ }^{\circ} 53$. The $90 \%$ confidence error circles of $C h a n d r a$ and VLT positions are also shown.

Table 1. VLA archival observations used in this paper.

\begin{tabular}{cccc}
\hline \hline Date & Project & \# of visibilities & Time on source \\
\hline 2003 Jul. 31 & AR523 & 249953 & $2807 \mathrm{~s}$ \\
2004 Oct. 16 & AR545 & 200182 & $1280 \mathrm{~s}$ \\
2006 Jan. 30 & AR570 & 151035 & $2153 \mathrm{~s}$ \\
\hline
\end{tabular}

as its counterpart. Furthermore, we report the first results of a follow-up observation with the Gran Telescopio de Canarias (GTC).

\section{GRS $1758-258$ revisited in the radio}

The National Radio Astronomy Observatory (NRAO) archive contains huge amounts of radio data from different sources that has accumulated over the years. Sometimes, several observations can be combined to produce a single deep radio map and reanalyzed with an aim different from the one it was originally planned. Similar approaches have been conducted by our group for X-ray binaries showing how useful archival data can be (see e. g. Sánchez-Sutil et al. 2008; Muñoz-Arjonilla et al. 2009).

In the context of this work, we explored the NRAO archive to improve the position of the core of GRS 1758-258. For this purpose, we restricted the selection criteria to those experiments carried out with the Very Large Array (VLA) in its extended A-configuration and with on-source times higher than a thousand seconds. Table 1 shows the observations used in this paper, all of them at the $6 \mathrm{~cm}$ wavelength. The total on-source time amounted to almost $2 \mathrm{~h}$. Observations were made in two IF pairs of $50 \mathrm{MHz}$ bandwidth each. The phase calibrator used was always 1751-253 which is located $2^{\circ}$ away from the target and has a position code $\mathrm{B}$ in the VLA Calibration Manual ${ }^{1}$. The data of each project were separately processed using the AIPS software package of NRAO following the standard procedures for continuum calibration of interferometers. All data

\footnotetext{
1 <http://www.vla.nrao.edu/astro/calib/manual/ csource.html>
}

sets were merged into a single $u v$ file, from which we computed our final map using natural weights. Radio contours corresponding to the core of GRS 1758-258 are shown in the right panel of Fig. 1. Our new and more accurate radio position, namely $\mathrm{RA}(\mathrm{J} 2000)=18^{\mathrm{h}} 01^{\mathrm{m}} 12^{\mathrm{s}} 40$ and $\operatorname{Dec}(\mathrm{J} 2000)=-25^{\circ} 44^{\prime} 36^{\prime \prime} 3$, was fitted in the image plane. Although it has a statistical error of $0 . \prime 04$ in both coordinates, we conservatively adopt an uncertainty of $0 . \prime 1$ to consider any possible systematics. Our declination value differs 0.5 from a previous result also derived with the VLA at $6 \mathrm{~cm}$ in the A-configuration and using the same calibrator source (Mirabel \& Rodríguez 1994). This apparent discrepancy may be caused by the position of the phase calibrator having been refined since those old observations, leading to a shift of nearly $0 . \prime 3$ almost completely in declination towards the south. Hence, our result represents an improvement of the radio position of the GRS 1758-258 core with respect to previous measurements (Mirabel \& Rodríguez 1994; Martí et al. 2002).

\section{The optical/near-infrared counterpart of GRS 1758-258}

\subsection{Reanalyzing archival data}

The improved radio position of GRS 1758-258, and the present day availability of modern star catalogues in the near-infrared, such as the Two Micron All Sky Survey (2MASS), motivated us to refine the old astrometry, which had been obtained in two steps because of the historic lack of suitable reference stars in the field (Martí et al. 1998). The total combined error in this old paper was close to $1^{\prime \prime}$, and a candidate counterpart could not be unambiguously proposed at that time.

Hence, we reanalyzed archival images at optical and nearinfrared wavelengths of the GRS 1758-258 field. In particular, we used images in the $R$ and $I$ bands obtained with the ESO New Technology Telescope (NTT) in 1998, and in the $K_{\mathrm{s}}$ band taken with the Very Large Telescope (VLT) in 2003 in a project by Heindl and collaborators. Astrometry was recalculated based on the data of more than 30 stars within our field 


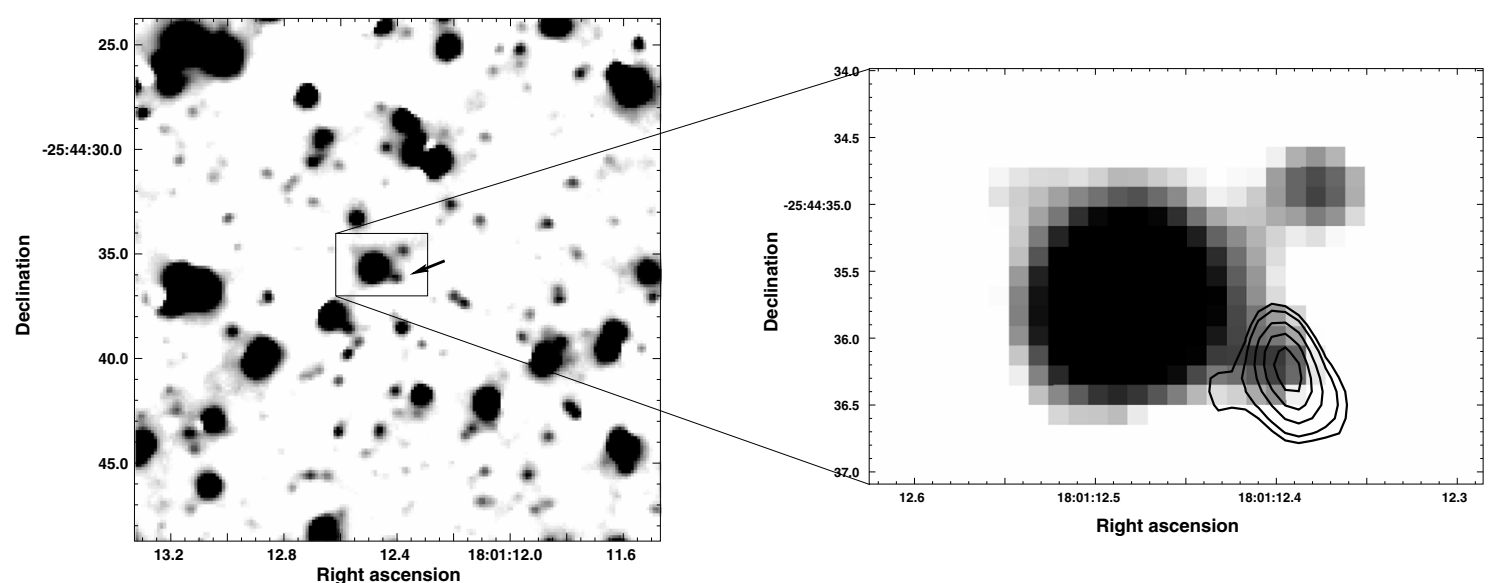

Fig. 2. Left. $K_{\mathrm{s}}$-band image corresponding to the central core of GRS 1758-258 taken with the VLT and the ISAAC instrument in 2003. The arrow points at the location of the near-infrared candidate counterpart. Right. Zoomed detail of the map on the left. Radio contours corresponding to the central core of GRS 1758-258 at $6 \mathrm{~cm}$ are shown, superimposed on the $K_{\mathrm{s}}$-band image. Contour levels are 4, 5, 7, 9, and 11 times the rms noise of $30 \mu \mathrm{Jy}$.

Table 2. Magnitudes of the candidate counterpart to GRS 1758-258.

\begin{tabular}{ccccc}
\hline \hline Filter & $\begin{array}{c}\text { Observation } \\
\text { date }\end{array}$ & $\begin{array}{c}\text { Apparent } \\
\text { magnitude }\end{array}$ & $\begin{array}{c}\text { Interstellar } \\
\text { extinction }\end{array}$ & $\begin{array}{c}\text { Dereddened } \\
\text { magnitude }\end{array}$ \\
\hline$R$ & 1998 Mar. 26 & $22.6 \pm 0.3^{a}$ & $6.3 \pm 0.5$ & $1.7 \pm 0.6$ \\
$I$ & 1998 Mar. 26 & $21.1 \pm 0.3^{a}$ & $4.0 \pm 0.3$ & $2.5 \pm 0.5$ \\
$K_{\mathrm{s}}$ & 2003 Aug. 23 & $16.9 \pm 0.2^{b}$ & $0.94 \pm 0.07$ & $1.3 \pm 0.3$ \\
\hline
\end{tabular}

Notes. ${ }^{(a)}$ Magnitude retrieved from a previous work (Martí et al. 1998); (b) magnitude computed in the present work.

whose accurate positions were retrieved from the 2MASS catalogue. The total combined astrometric error is 0 .' 16 for the NTT maps and 0.' 18 for the VLT image. As a result, among the three candidate counterparts of GRS $1758-258$ that were proposed almost a decade ago, only one of them remains consistent with both the VLA and Chandra positions (see Figs. 1 and 2 and their right panel zooms). Approximate photometry was calculated in the $K_{\mathrm{s}}$ image by estimating the zero point after comparing the instrumental magnitudes of ten stars in the field with the corresponding ones in the 2MASS catalogue. The $K_{\mathrm{s}}$ magnitude derived here for GRS $1758-258$ is clearly consistent with previous measurements (Eikenberry et al. 2001). Magnitudes in the $R$ and $I$ bands were directly retrieved from the original work (Martí et al. 1998). These apparent magnitudes are listed in Table 2.

\subsection{GTC follow-up observations}

We carried out spectroscopic observations using the $10 \mathrm{~m} \mathrm{GTC}$ and the OSIRIS instrument ${ }^{2}$ at the Observatorio de La Palma (Spain) in long-slit mode. Our goal was to obtain a spectrum of the optical candidate counterpart of GRS 1758-258, and the GTC with OSIRIS in a low-resolution spectral mode seemed an appropriate combination to use for such a weak source. The idea was to identify any absorption or emission feature that could allow us to discriminate between a Galactic or an extragalactic origin for this counterpart of GRS 1758-258, and therefore to know if we should carry on considering it as an archetypical Galactic microquasar.

\footnotetext{
$2<$ http://www.iac.es/project/OSIRIS/>
}

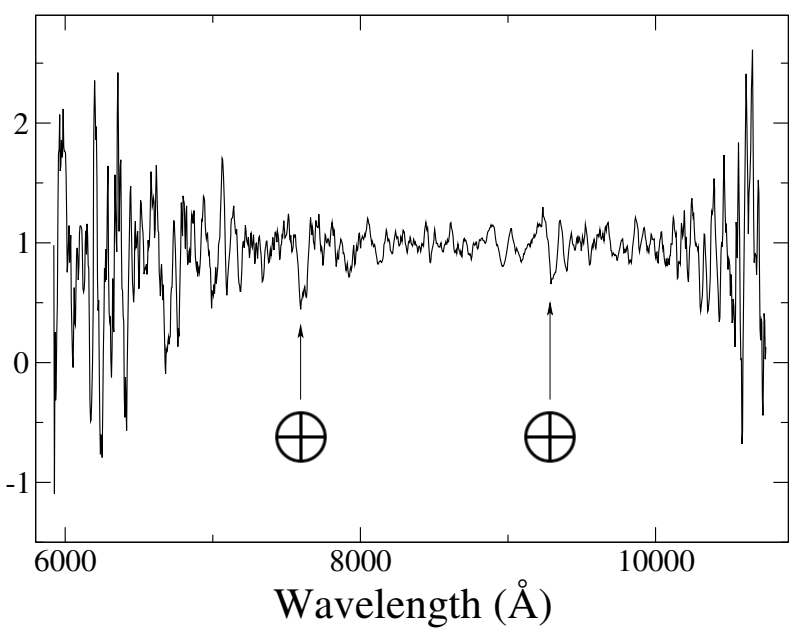

Fig. 3. Noisy spectrum of the proposed GRS 1758-258 optical counterpart. Only telluric features ( $\oplus$ in the figure) are obvious.

The observations were performed on May 21 and June 30 2009. The slit was aligned with the parallactic angle to minimize light loss due to atmospheric refraction. The grism covered the 3700-10000 $\AA$ spectral range, and the integration time amounted to $2.3 \mathrm{~h}$. All frames were reduced using standard procedures for sky background subtraction, flat-fielding, and extraction of the spectrum, based on the IRAF ${ }^{3}$ image processing system. Unfortunately, the seeing conditions, the weakness of our target, and our high air-mass value even at transit time rendered the observation very difficult. Figure 3 shows the resulting noisy spectrum, after wavelength calibration and continuum normalization. The shortest wavelengths covered by the grism are not shown since no signal is detected above the noise.

\section{Discussion}

Figure 2 again shows the GRS 1758-258 radio map superimposed on the VLT $K_{\mathrm{s}}$-band image. The proposed optical/nearinfrared candidate counterpart, which is the only one that is astrometrically coincident with Chandra and VLA positions of the core of GRS $1758-258$, is clearly seen. It turns out to be

$3<$ http://iraf.noao.edu/> 
a weak and highly absorbed source that is barely detected towards the blue. The offsets from the improved radio position are $\Delta \alpha \cos \delta \simeq 0$.' 16 and $\Delta \delta \simeq 0$.'14, which are clearly consistent with astrometric errors.

The GTC spectrum of the candidate counterpart of GRS 1758-258 shown in Fig. 3 is probably also contaminated by those of the two stars that are just $\sim 1^{\prime \prime}$ away. Apart from some telluric features, no spectral lines were confidently observed. Nevertheless, the lack of strong emission lines, which would be visible even in a contaminated spectrum if GRS 1758-258 were a sort of nearby active galaxy (e. g., a Seyfert or a FR-II), is consistent with the usual interpretation of GRS $1758-258$ as a source within the Galaxy. However, in this case, the eccentricity of the orbit of the star would need to be very high to fill its Roche lobe if a 18.45 d period were assumed (Rothstein et al. 2002; Smith et al. 2002). The problems raised by this inconsistency will require further attention to unveil the true physical scenario behind GRS 1758-258.

In the absence of conclusive spectroscopic data, only broad-band photometry can place broad constraints on the physical nature of GRS 1758-258. An interstellar extinction of $A_{\mathrm{V}} \simeq 8.4$ mag was estimated following a similar approach to that of Predehl \& Schmitt (1995) and considering a column density towards GRS 1758-258 of $N_{\mathrm{H}} \simeq(1.5 \pm 0.1) \times 10^{22} \mathrm{~cm}^{-2}$ (Mereghetti et al. 1997). We also computed the values $A_{K}, A_{I}$, and $A_{R}$ using the relations reported by Rieke \& Lebofsky (1985). Assuming a Galactic center distance of $8.5 \mathrm{kpc}$, dereddened magnitudes of the candidate counterpart to GRS 1758-258 are obtained. The results of our final revised photometry are summarized in Table 2. We searched for any possible variability of the proposed counterpart in the ESO archives. A total of 11 observing nights of data were available, obtained with VLT and the ISAAC instrument in the $K_{\mathrm{s}}$-band. No variability was detected with amplitude larger than $\pm 0.5 \mathrm{mag}$ on a timescale of weeks. The $K_{\mathrm{s}}$ dereddened magnitude is roughly consistent with an early A-type main sequence star. However, the corresponding colours $(R-I) \simeq-0.8$ and $(I-K) \simeq+1.2$ are inconsistent with this or any other spectral type. In a Galactic context, this may be indicative of the optical/near-infrared luminosity being dominated by a non-stellar component (e. g., an accretion disk) as we would expect from a low-mass X-ray binary. On the other hand, we cannot rule out the possibility that this might be caused by the brighter star $\left(K_{\mathrm{s}} \simeq 13.7\right)$, which is located very close to our proposed candidate counterpart and may contaminate the photometry even after we have carefully tried to subtract its effects. Since a different extinction law may explain the discrepant colours, we computed them again following the conversions of near-infrared extinctions to $A_{V}$ towards the nuclear bulge described by Gosling et al. (2009). Despite the different form of the extinction law, the derived colours do not substantially change compared to those listed in Table 2. Hence, our conclusion of contamination by either the nearby star or by a non-stellar component such as an accretion disk remains unaltered.

Despite the challenge to interpret spectroscopic observations, the main contribution of this work has been the identification of a serious candidate to the optical/near-infrared counterpart of GRS 1758-258 based on accurate astrometric coincidence. This opens the possibility of investigating the true nature of this source. Additional spectroscopic observations with $8 \mathrm{~m}$-class telescopes and adaptive optics at southern locations are required to confirm its Galactic (or extragalactic) origin and, in the former case, to more clearly classify its spectral type.

Acknowledgements. The authors acknowledge support by grant AYA200768034-C03-02 from the Spanish government, and FEDER funds. This has been also supported by Plan Andaluz de Investigación, Desarrollo e Innovación of Junta de Andalucía as research group FQM322 and excellence fund FQM5418. J.A.C. is a research member of the Consejo Nacional de Investigaciones Científicas y Tecnológicas (CONICET), Argentina. The NRAO is a facility of the NSF operated under cooperative agreement by Associated Universities, Inc. This publication makes use of data products from the Two Micron All Sky Survey, which is a joint project of the University of Massachusetts and the Infrared Processing and Analysis Center/California Institute of Technology, funded by the National Aeronautics and Space Administration and the National Science Foundation in the USA. This paper is based on observations made with ESO Telescopes at the La Silla Paranal Observatory under programme ID $<71$.D-0387>. This paper is also based on observations collected with the Gran Telescopio de Canarias (GTC) at Observatorio del Roque de los Muchachos (La Palma, Canary Islands) operated by the Instituto de Astrofísica de Canarias (IAC). We also thank the anonymous referee, whose constructive comments helped us to significatively improve this paper.

\section{References}

Eikenberry, S. S., Fischer, W. J., Egami, E., \& Djorgovski, S. G. 2001, ApJ, 556, 1

Goldwurm, A., Cordier, B., Paul, J., et al. 1994, Nature, 371, 589

Gosling, A. J., Bandyopadhyay, R. M., \& Blundell, K. M. 2009, MNRAS, 394, 2247

Heindl, W. A., \& Smith, D. M. 2002, ApJ, 578, L125

Martí, J., Mereghetti, S., Chaty, S. et al. 1998, A\&A, 338, L95

Martí, J., Mirabel, I. F., Rodríguez, L. F., \& Smith, I. A. 2002, A\&A, 386, 571

Mereghetti, S., Cremonesi, D., Haardt, F., et al. 1997, ApJ, 476, 829

Mirabel, I. F., \& Rodríguez, L. F. 1994, AIPC, 304, 413

Mirabel, I. F., \& Rodríguez, L. F. 1999, ARA\&A, 37, 409

Mirabel, I. F., Rodríguez, L. F., Cordier, B., Paul, J., \& Lebrun, F. 1992, Nature, 358,215

Muñoz-Arjonilla, A. J., Martí, J., Combi, J. A., et al. 2009, A\&A, 497, 457

Predehl, P., \& Schmitt, J. 1995, A\&A, 293, 889

Rieke, G., \& Lebofsky, M. 1985, ApJ, 288, 618

Rodríguez, L. F., Mirabel, I. F., \& Martí, J. 1992, ApJ, 401, L15

Rothstein, D. M., Eikenberry, S. S., Chatterjee, S. et al. 2002, ApJ, 580, L61

Sánchez-Sutil, J. R., Martí, J., Combi, J. A., et al. 2008, A\&A, 479, 523

Smith, D. M., Heindl, W. A., \& Swank, J. H. 2002, ApJ, 578, L129

Sunyaev, R., Churazov, E., Gilfanov, M., et al. 1991, A\&A, 247, L29 